\title{
Differences in Skin Tone among Hispanic Inmates in Florida's Prisons
}

\section{by Carmen M. Cusack, J.D., Ph.D. \\ The author wishes to acknowledge and thank $R C$ and $Y C$.}

\author{
Abstract \\ Purpose: This research asked whether darker skin tones are more prevalent among Hispanic inmates \\ in Florida.
}

\begin{abstract}
Methods: Von Luschan's Chromatic Scale includes 36 shades between "white" and "black". Raters compared photos of inmates to the shade scale.
\end{abstract}

\begin{abstract}
Results: Among female inmates in Florida, "brown" shades were most abundant. "Brown" shades are darker than intermediate or "olive" shades, which are reportedly most abundant among Hispanics worldwide. However, "black" shades were not as prevalent among female inmates. Among male inmates, "black" and "brown" shades were most abundant. Proportionally, more female inmates than male inmates were light.
\end{abstract}

Conclusion: These findings are potentially relevant to literature about differences between Whites, Blacks, and Hispanics in arrests, sentencing, and incarceration.

Key words: shade, inmates, Florida, Hispanics, White, Black, Native, Mestizo, dark-skinned, lightskinned, olive-skinned, brown people, racism.

\section{Introduction}

Differences between Whites, Blacks, and Hispanics within the criminal justice system are well documented by researchers, the U.S. Department of Justice, and numerous advocacy groups. The first difference is that arrest rates are higher among Blacks and Hispanics. The second difference is that Whites invest in quality legal representation and Blacks and Hispanics do not. Judges tend to sentence Blacks and Hispanics more harshly, and Whites are favoured by systemic processes. Hispanics' ethnicity may be identifiable by ethnic names or perhaps appearance. Some Hispanics may not immediately appear to be Hispanic because their features or complexion seem to pertain to White or Black races. Throughout Latin American cultures, racism against Natives and Blacks is not uncommon, and fairer skin has been associated with upper classes. In the U.S. Hispanics range in skin tone from "white" to "black". However, if Blacks and Hispanics are arrested more frequently and experience other differences within the criminal justice system, then perhaps darker Hispanics would be more prevalent in prisons than lighter Hispanics. Darker Hispanics might possibly be grouped, by society or the system, with Blacks, whereas lighter Hispanics might experience similar privileges to those granted to Whites, especially if they "pass" as or identify as White. It would follow that incarcerated Hispanics would tend to have darker skin tones.

\section{Literature Review}

Differences among Whites, Blacks, and Hispanics in the U.S. criminal justice system are well documented. Mauer and King reported that in 2005, the population of Hispanic inmates had risen $43 \%$ over the past 15 years (Mauer \& King 2007). Hispanics were $20 \%$ of state and federal inmates (Maver \& King 2007). In general, $1 \%$ of the population is incarcerated in the U.S. (NIJ 2012). In the U.S. $2.3 \%$ of Blacks, $0.4 \%$ of Whites, and $0.7 \%$ of Hispanics are incarcerated (Mauer \& King 2007). In seven states, more than $1 \%$ of Hispanics are incarcerated (Maver \& King 2007). Approximately $16 \%$ of Hispanic males born in 2007 will be incarcerated (Maver \& King 2007). For Hispanic females, 
the number is much lower; only about $2 \%$ of Hispanic females born in 2007 will be incarcerated (Maver \& King 2007). This is twice the rate of White men and women (Maver \& King 2007). Only 412 of every 100,000 Whites are incarcerated, but 2,290 Blacks and 742 Hispanics of every 100,000 are incarcerated. Disproportionality is highest in Massachusetts, New Hampshire, New Jersey, New York, and Pennsylvania state penitentiaries (Maver \& King 2007). More Whites are incarcerated in Oklahoma than any other state, but in 16 states Hispanics are incarcerated at higher rates (Mauer \& King 2007). Unfortunately, about one-quarter of states do not distinguish Hispanics from Whites or Blacks in their records (Maver \& King 2007). Researchers suspect that White and Black categories also absorb some Hispanic inmates in prisons that differentiate between the three groups or when inmates are White-Hispanic or Black-Hispanic mixes (Maver \& King 2007).

Among all federal offenders, Hispanics were more likely than non-Hispanics to be incarcerated (Lopez \& Light 2009). Because most federal crimes committed by Hispanics were immigration offences, Hispanics usually received shorter sentences than Whites or Blacks (Lopez \& Light 2009). Approximately one third of federal inmates are Hispanic and one third are White (Lopez \& Light 2009). Nearly forty percent are Black (Lopez \& Light 2009). However, fewer than $10 \%$ of all people incarcerated in the U.S. are in federal penitentiaries; the great majority of the 2.3 million inmates serve time in state prisons (Lopez \& Light 2009). Almost one-fifth of state inmates are Hispanic, more than one-third are White and approximately 40\% are Black (Lopez \& Light 2009). The numbers are similar in local jails (Lopez \& Light 2009).

The percentage of Hispanics who are sentenced is even higher than those incarcerated (Lopez \& Light 2009). In 2007, 40\% of all offenders sentenced for federal crimes were Hispanic (Lopez \& Light 2009). Almost three-quarters of Hispanics sentenced in federal court in 2007 were not U.S. citizens (Lopez \& Light 2009). The great majority of these and all offenders are men (NIJ 2012). Two percent of all men in the U.S. are incarcerated, but fewer than .05\% of women (NIJ 2012). More than 10\% percent of Black men in their 205 and early 305 are incarcerated and Black women are incarcerated at the same rate as the national average, $1 \%$ (NIJ 2012).

Racial differences in the criminal justice system stand in severe contrast to the fact that Whites commit many unpunished crimes (Kansal 2005). For example, approximately $7 \%$ of White and Black community members use drugs monthly (Kansal 2005). Around 6\% of Hispanic community members use drugs monthly (Kansal 2005). Thus, White community members comprise about $72 \%$ of monthly drug users in the U.S. (Kansal 2005). Hispanics are around $11 \%$ and Blacks are $13 \%$ of monthly drug users (Kansal 2005). The great majority of these community members buy drugs from people of their own race, which means that the bulk of drug sales in the U.S. are between Whites (Kansal 2005).

Whites who provide prosecutors with assistance receive greater sentence reductions than Blacks or Hispanics (Kansal 2005). Black and Hispanic recidivists who commit serious crimes are punished more harshly than similarly situated Whites (Kansal 2005). Hispanics in Miami who recidivated were sentenced more harshly than Whites and were more likely to be incarcerated (Kansal 2005). Even less serious crimes were punished more severely among Hispanics and Blacks (Kansal 2005). More than Hispanics or Whites, Blacks are more typically jailed pending trial, which leads to harsher sentencing (Kansal 2005).

Wealth and poverty likely contribute to differences between Blacks, Whites, and Hispanics. For example, more than Hispanics or Blacks, Whites tend to invest in private representation, which correlates with more favorable dispositions (Kansal 2005). These differences do not necessarily emerge within the justice system. Racism related to race, shade, and class within White, Hispanic, and Black communities is also prevalent (Padilla 2001). For example, Blacks may experience racial tension due to distinct shades, e.g. "Ol' Whitey/ Uncle Toms", "light-skinned", "high yellow", "red", 
"crispy", and "coal-miner" (Manjarres 2013). Lighter tones are rooted in interraciality and communities sometimes link shade with status (Manjarres 2013). Whites also exhibit some racial tension relating to status (Cusack 2013). For example, Whites disparage classless people as "red necks", a term related to tans acquired during fieldwork. Whites also use the term "white trash" to racially identify certain economically disadvantaged and classless people. Among Hispanics, lighter skin has been given higher status in some communities. Blacks, who are believed to be slave descendants, and "brown"toned or "red"- toned people, who are believed to be Mestizos or of native descent, sometimes experience racism from other Hispanics (HDRC 2001). Blacks and Mestizos are often less wealthy than Hispanics with greater proximity to European descendency (HDRC 2001). For example, one can compare Hispanics in Argentina with Nicaraguans and Guatemalans (HDRC, 2001; Navarro, 2012). White Hispanics are sometimes perceived as being wealthy (Sailer 2000). For example, blue-eyed and fair-skinned Mexicans are somewhat rare and are frequently wealthier members of the population (Sailer 2000).

Mexico's white elite finds life south of the border far too sweet to come north for anything other than advanced degrees, advanced medical care, and advanced shopping. The CIA Factbook claims that Mexico's 100 million people are $9 \%$ white, 60\% mestizo, 30\% Indian, and $1 \%$ other.... Since Spaniards and Indians tend to share dark hair and dark eyes, and aren't all that far apart in skin color, without DNA tests it's hard tell whether or not people who look pure Spanish or Indian aren't really slightly mixed.... Nonetheless, the Mexican elite tends to look strikingly European (Sailer 2000).

When poorer, darker Hispanics enter the U.S. illegally and commit crimes, then they may not receive any support from resident Hispanics or citizens or be able to afford representation (Kansal, 2005; Lopez \& Light, 2007). Even when naturalized, stigmatization and exclusion may continue within their cultures or society at large (Lopez \& Light 2009).

Hispanics may feel that Hispanic is a separate race than White or Black, though more than two-thirds of U.S. census-takers in 2012 identified themselves as pertaining to a race, e.g. Native, Black or White (Navarro 2012). For example, $2.5 \%$ of Hispanics identify as racially Black (Navarro 2012). Of these $2.5 \%$ of Hispanics, many feel that they are treated prejudicially due to their color and appearance as Black (Navarro 2012). Some lighter Hispanics identify as White or try to "pass" as White (Navarro, 2012; Padilla, 2001). This may be due to a preference for White privilege, affinity with White culture, or an embedded rejection of Blacks in Hispanic culture (Pew Research 2008). Thus, shade among Hispanics may be a source of tension at community levels as well as a determining factor within the criminal justice system.

\section{Methods}

This research asks whether certain skin tones are more prevalent among Hispanic inmates in Florida. The null hypothesis was that every skin tone would be equally prevalent among Hispanics in prison. The researcher expected that the skin tones most prevalent among Hispanics in the general population ought to be most prevalent, yet hypothesized that darker skin tones would be most prevalent (see Figure 1 below).

Among Floridian inmates, a randomized convenience sample was used because race records including a "Hispanic" category were available online with photos, and the State of Florida is populated by Hispanics from different countries, including Cuba, Puerto Rico, Mexico, and Nicaragua, which are 
nations comprised of different mixes of European, African, and Native heritage (Vogel, 2013).

Von Luschan's Chromatic Scale was used to classify inmates' shades (see Figure 1). Von Luschan's is a shade scale featuring 36 distinct shades. The scale is made of the following six parts, which each contain six shades: 1) Very light or white, "Celtic"; 2) Light/ light European; 3) Light intermediate/ dark European; 4) Dark intermediate/ "olive skin"; 5) Dark/ "brown"; and 6) Very dark/ "black". The scale was originally made of tiles that were held to a person's skin, usually the inside of the arm. However, a two dimensional scale is used on countless websites now. Reliability was ensured by using standard Tru Color 32 bit screen color.

Figure 1: Von Luschan's Chromatic Color Scale

\begin{tabular}{|l|l|l|l|l|l|l|l|}
\hline & 1 & 10 & & & 19 & 28 \\
\hline & 2 & 11 & & & 20 & 29 \\
\hline 3 & 12 & & & 21 & 30 \\
\hline & 4 & 13 & & & 22 & 31 \\
\hline & 5 & 14 & & & 23 & 32 \\
\hline & 6 & 15 & & & 24 & 33 \\
\hline & 7 & 16 & & & 25 & 34 \\
\hline & 8 & 17 & & & 26 & 35 \\
\hline & 9 & 18 & & & 27 & 36 \\
\hline
\end{tabular}

Based on ample anecdotal evidence found throughout the web, it seems that most Hispanics are considered to be or identify as shades III and IV, light intermediate or "olive". (see Figure 2 below). The Fitzpatrick Test is another skin tone test that groups complexions into four categories (Melanoma Foundation 2013). Rather than separate intermediate, "olive", and "brown" skin tones, it collapses the group into a single category, Type III (Melanoma Foundation 2013). Arguably, the majority of Hispanics separated into different groups on Von Luschan's scale would fit into Type III (Melanoma Foundation 2013). Though, the Fitzpatrick Test separates darker and Black Hispanics into a distinct group, Type IV (Melanoma Foundation 2013). This is why Von Luschan's scale was ideal for this study. 


\section{Figure 2: Von Luschan Chromatic Color Scale, Human Skin Color Distribution}

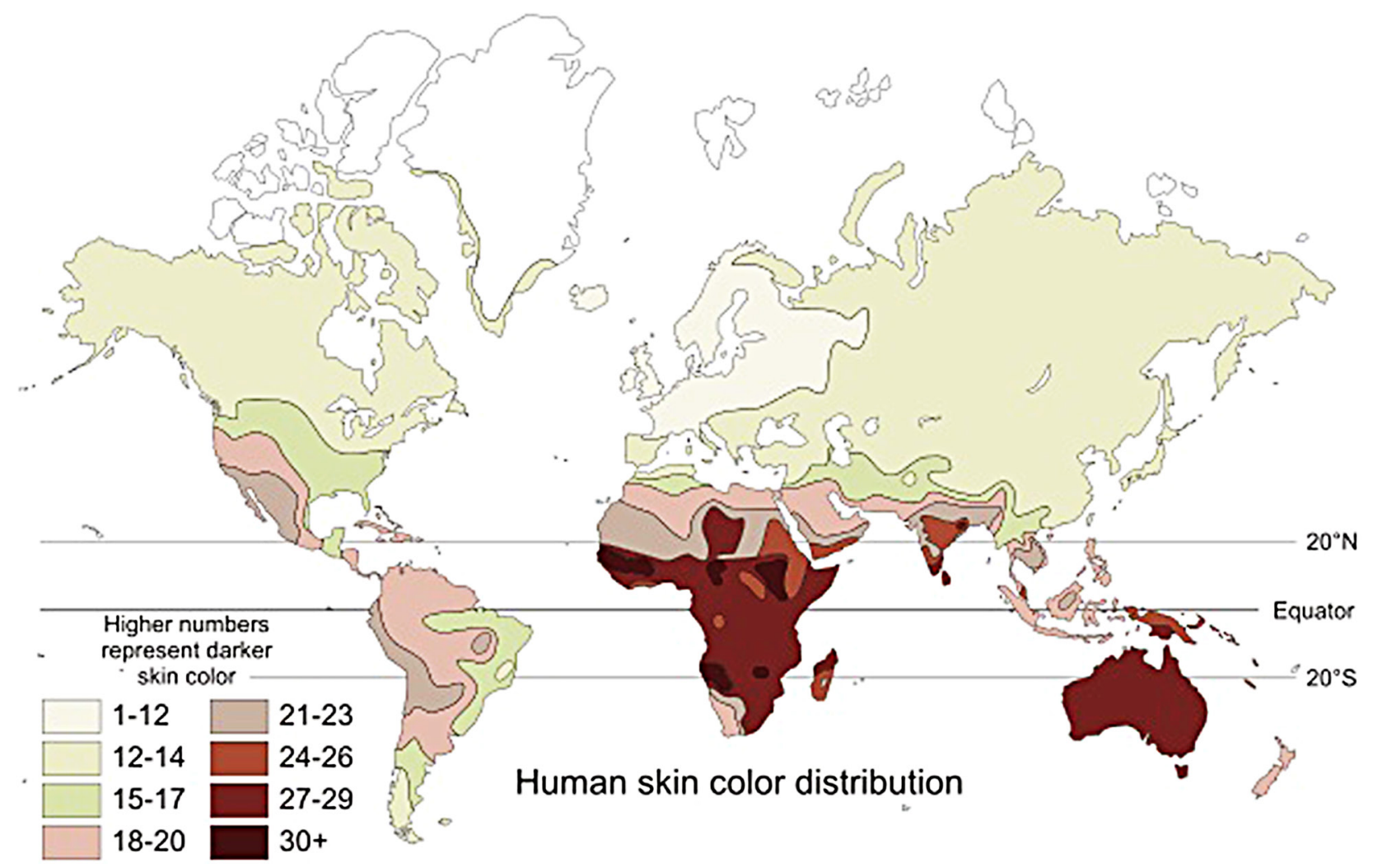

The Department of Corrections in Florida displayed photos of 3,764 Hispanic males and females incarcerated in Florida on November 11, 2013. Only 164 female inmates were incarcerated. Of these, 100 were randomly included. Males were more prevalent with 3,600 inmates. Of these, 1,000 were randomly included. Two raters viewed the photos and a third rater voted to break conflicting classifications. When the third rater chose a third shade not chosen by the first two raters, the photo was eliminated from the study. No identifying information, including names or crimes committed, was recorded. Gender and shade were recorded and analyzed using Microsoft Excel.

\section{Findings}

The following are the number of female inmates for each shade: Type I: 1) $n=0,2) n=2,3) n=0 ; 4) n=0$; 5) $n=0$; Type II: 6) $n=0$; 7) $n=0$; 8) $n=3$; 9) 0; 10) $n=0$; Type III: 11) $n=0$, 12) $n=1$, 13) $n=0,14) n=2$, 15) $n=1$; Type IV: 16) $n=2$, 17) $n=1$, 18) $n=4$, 19) $n=4$, 20) $n=2$; 21) $n=4$; Type $V: 22) n=3$, 23) $n=2,24) n=16,25) n=8$, 26) $n=6,27) n=8,28) n=15$; Type VI: 29) $n=5,30) n=6,31) n=2,32) n=0,33) n=3$.

The following are the number of male inmates for each shade: Type I: 1) $n=1,2) n=2,3) n=1 ; 4) n=0$; ) $n=1$; Type II: 6) $n=0$; 7) $n=0$; 8) $n=3$; 9) 0; 10) $n=0$; Type III: 11) $n=0,12) n=4$, 13) $n=4$, 14) $n=0$, 15) $n=20$; Type IV: 16) $n=5$, 17) $n=10,18) n=11,19) n=12,20) n=37$; 21) $n=56$; Type $V: 42) n=42$, 23) $n=17,24) n=48$, 25) $n=76$, 26) $n=116$, 27) $n=74$, 28) $n=91$; Type VI: 29) $n=37$, 30) $n=89$, 31) $n=75$, 32) $n=26,33) n=32$, 34) $n=15,35) n-94,36) n=1$.

Only two females and five males were rated as very light, Type I. Four females and 28 males were light, Type II. In Type III, intermediate, 17 female inmates and 131 male inmates were identified. Females in Type IV, "brown", numbered 58 and males numbered 464. "Black" female inmates in Type $\checkmark$ numbered 16 and males number 369 . 


\section{Analysis}

Only five photos were eliminated due to dispute about inmates' shade. Inter-rater reliability was .95. Among the sample, $2 \%$ of females but only . $5 \%$ of males were very light, Type I. Four percent of females were light, and almost 3\% of men were light, Type II. In Type III, "olive" skin, 17\% of females and approximately $13 \%$ males were incarcerated. Almost $60 \%$ of all incarcerated females were "brown". Forty-six percent of males were Type IV. "Black" type women were $16 \%$ of the females sampled, and nearly $40 \%$ of males sampled were "black". For females, the 10 most prevalent shades, which is about one third of all possible shades, amounted to 12 "olive", 11 "black", and 53 "brown". Among male inmates, 56 inmates were shades of "olive", 258 were shades of "black" and 447 were shades of "brown".

\section{Discussion}

Since two females and five males were rated in Level I, it means that $2 \%$ of females but only $.5 \%$ of males were very light, which perhaps indicates that very light Hispanic males are incarcerated at lower rates in Florida. Maybe they are classified by the system as White, not Hispanic. It seems that if they are incarcerated at lower rates then further research about arrest and sentencing of very light Hispanics is necessary. Because of the difference between these rates and national averages, it seems that to some extent, very light Hispanic males are more privileged than very light Hispanic women. This trend should be investigated in federal penitentiaries where most Hispanics committed immigration violations. No doubt some if these inmates were targeted by authorities because they appeared to be Hispanic. Perhaps very light Hispanics are targeted less frequently by federal authorities investigating suspected immigration violations.

Light Hispanic males and females were much closer proportionally. The number was quite low. Explanations could include lower proportion of light Hispanics in general or in Florida, the Sunshine State. Other explanations might include labeling inmates as White, or a tendency for fewer light Hispanics to be incarcerated. Again, in Type III, women have higher rates. This finding supports the trend in Type I that Hispanic females on the lower end of Von Luschan's scale are incarcerated in Florida at higher rates than Hispanic males.

Type IV was most prevalent among females. This skin tone is common to the western coast of South America, Southeast Asia, Africa, and Australia (see Figure 2). It is not the predominant shade in Latin America, but seems to predominate in countries with higher Mestizo mixes. Fewer males than females were "brown" but Type IV comprised almost half of all Hispanic male inmates. This result is interesting because most studies show low rates for incarceration of Hispanic females but high rates for males. Yet, the bulk of both groups was comprised of "brown" people. Thus, between males and females, which experience severely different incarceration rates, "brown" shades were most frequent among both groups. "Black" type females were slightly less numerous than "olive" females. Clearly, "brown" comprised the great majority of inmates. Arrest rates, counsel, convictions, and sentencing could potentially correlate with Blacks and Hispanics, so perhaps one factor for this among Hispanic women is "brown" complexion.

Either because "brown" skin flags females as Hispanic or possibly because it groups them more closely with Blacks, but further from Whites, shade may play a role. Von Luschan's scale indicates that "black" tones are not common in Latin America. They are mainly found in Australia and Africa. Yet, almost half of Hispanic males incarcerated in Florida were "black". This trend is supported by literature showing higher incarceration rates for Blacks and Hispanics. These results are not necessarily generalizable because Florida is a sunny state, yet they open the door to further inquiry about the relationship between skin tone among Hispanics and the criminal justice system. 


\section{Limitations}

The number of Black-Hispanic, White-Hispanic, or Mestizo inmates is not known. This information would have added robustness to this study. Inmates' photos may not be the same shade as when they were arrested or sentenced. For example, spending an hour in the prison courtyard each day or 23-24 hours in-doors could change an inmate's shade from the time arrest or sentencing occurred. Inmates serving long sentences may not be the same shade as when they were arrested or sentenced, but could be photographed at any point before appearing before a parole board. Shades may have been impacted by poor lighting conditions during photography, yet the relationship between shades is still evident. In addition to lighting conditions in the room or camera, other factors could have temporarily altered inmates' shades. Factors could include alcohol, food or drug consumption, physical contact or irritation, emotion, temperature, allergies, or exertion.

One limitation of the Von Luschan scale is that it lacks variety in red and orange tones. Though it offers many yellowish, golden, and brown tones, reddish tones were not varied enough to precisely match each inmate. This study would have benefited from a scale that included greater variation among red shades within the "brown" tones. The chart is intended to be used on the inside of the arm, where skin has been less exposed to sunlight, however, here it is used on the face, which is often most exposed to sun. Thus, shades here may not necessarily be comparable with findings in studies using the scale only on inner arms. Finally, human skin is rich with tones. No face is monochromatic. Thus, shades identified here identified the predominate shade.

\section{Implications for Future Study and Conclusion}

The relationship between race and the criminal justice system is complex and often disproportionally severe against Hispanics and Blacks. Hispanics who "pass" or identify as White may receive some lenience, however, those who are affiliated with Blacks, due to race, shade, or perhaps Native heritage, may encounter greater difficulties than "white" or light Hispanics. This study indicated that "brown" and "black" Hispanics comprise the bulk of inmates in Florida. Yet, these shades are not proportional to the general population of Hispanics.

This trend should be investigated further and among other populations, e.g. Natives and Blacks. Lighter Hispanic males were not a populous category in Florida prisons, yet females were proportionally much higher. These rates should be studied further and similar studies should be conducted among other light-skinned populations, e.g. Blacks or Whites. Findings here indicated that there is a relationship between darkness and incarceration in Florida. Thus, other state and federal inmates should be studied and compared. These findings can serve the criminal justice system by highlighting the potential significance that shade plays within races and the effect that it could have on the disposition of cases. 


\section{References}

Human Rights Documentation Center (HDRC). (2001). Executive Summary, Racial Discrimination: The Record of Argentina. Retrieved from http://academic.udayton.edu/race/o6hrights/georegions/ southamerica/argentina01.htm

Pew Research. (2008). Do Blacks and Hispanics Get Along? Yes, but Not Always, and Not about Everything. Retrieved from http://www.pewsocialtrends.org/2008/01/31/do-blacks-and-hispanicsget-along/

Cusack, C. M. (2013). Irish in the Criminal Justice System. Journal of Law \& Social Deviance. 6. 1-29.

Kansal, T. (2005). Racial Disparity in Sentencing: A Review of the Literature. The Sentencing Project. Retrieved from http://www.sentencingproject.org/doc/publications/rd_sentencing_review.pdf

Lopez, M.H. \& Light, M. (2009, Feb. 18). A Rising Share: Hispanics and Federal Crime. Pew Research Hispanic Trends Project. Retrieved from http://www.pewhispanic.org/2009/02/18/a-rising-sharehispanics-and-federal-crime/

Manjarres, J. (2013, Jul. 19). The Hispanic Color Wheel, From White to Brown. SharkTank. http://sharktank.net/2013/07/19/the-hispanic-color-wheel-from-white-to-brown/\#sthash.JoSX7t1L.dpuf

Mauer, M. \& King, R.S. (2007). Uneven Justice: State Rates of Incarceration by Race and Ethnicity. The Sentencing Project. Retrieved from http://www.sentencingproject.org/doc/publications/rd_ stateratesofincbyraceandethnicity.pdf

National Institute of Justice (NIJ). (2012, June 4). Figure 2. U.S. Incarceration Rates by Race and Sex. Retrieved from http://www.nij.gov/journals/270/criminal-records-figure2.htm

Navarro, M. (2012, January 13). For Many Latinos, Racial Identity Is More Culture Than Color. The New York Times. Retrieved from http://www.nytimes.com/2012/01/14/us/for-many-latinos-race-is-moreculture-than-color.html?_r=o

Padilla, L. M. (2001). But You're Not a Dirty Mexican: Internalized Oppression, Latinos and Law. Texas Hispanic Journal of Law and Policy. 7, 61.

Sailer, S. (2000, May 12). Importing Mexico's Worsening Racial Inequality. VDare. Retrieved from http://www.vdare.com/articles/importing-mexicos-worsening-racial-inequality

Vogel, M. (2013, April 30). Snapshots of Florida's Hispanic Community. Florida Trend. Retrieved from http://www.floridatrend.com/article/15521/snapshots-of-floridas-hispanic-community?page=1

Melanoma Foundation (2013). Which Type of Skin Do You Have? Viewed on Nov. 15, 2013. Retrieved from http://www.melanomafoundation.com/pdf/Skin\%2oTypes.pdf 